\title{
Nervous Wreck and Cdc42 Cooperate to Regulate Endocytic Actin Assembly during Synaptic Growth
}

\author{
Avital A. Rodal, Rebecca N. Motola-Barnes, and J. Troy Littleton \\ The Picower Institute for Learning and Memory, and Departments of Biology and Brain and Cognitive Sciences, Massachusetts Institute of Technology, \\ Cambridge, Massachusetts 02139
}

\begin{abstract}
Regulation of synaptic morphology depends on endocytosis of activated growth signal receptors, but the mechanisms regulating this membrane-trafficking event are unclear. Actin polymerization mediated by Wiskott-Aldrich syndrome protein (WASp) and the actinrelated protein $2 / 3$ complex generates forces at multiple stages of endocytosis. FCH-BIN amphiphysin RVS (F-BAR)/SH3 domain proteins play key roles in this process by coordinating membrane deformation with WASp-dependent actin polymerization. However, it is not known how other WASp ligands, such as the small GTPase Cdc42, coordinate with F-BAR/SH3 proteins to regulate actin polymerization at membranes. Nervous Wreck (Nwk) is a conserved neuronal F-BAR/SH3 protein that localizes to periactive zones at the Drosophila larval neuromuscular junction (NMJ) and is required for regulation of synaptic growth via bone morphogenic protein signaling. Here, we show that Nwk interacts with the endocytic proteins dynamin and Dap160 and functions together with Cdc42 to promote WASp-mediated actin polymerization in vitro and to regulate synaptic growth in vivo. Cdc42 function is associated with Rab11-dependent recycling endosomes, and we show that Rab11 colocalizes with Nwk at the NMJ. Together, our results suggest that synaptic growth activated by growth factor signaling is controlled at an endosomal compartment via coordinated Nwk and Cdc42-dependent actin assembly.
\end{abstract}

Key words: actin; endocytosis; F-BAR; Drosophila; neuromuscular junction; synaptic growth

\section{Introduction}

In response to changes in activity, neurons exhibit structural plasticity that contributes to long-lasting alterations in neuronal connectivity. However, the specific mechanisms by which activity-dependent cues are translated into morphological change remain unclear. The Drosophila neuromuscular junction (NMJ) serves as a useful model for characterizing these signaling pathways. During larval development, the muscle surface area grows 100 -fold, requiring elevated synaptic input to achieve muscle membrane depolarization. To maintain synaptic strength, synaptic arbors expand by adding new boutons, resulting in a stereotyped number of contacts per unit of muscle surface area. The mechanisms regulating this growth involve neuronal activity, retrograde signals from the muscle, and anterograde signals from neuron to muscle (Collins and Diantonio, 2007).

Mutations disrupting endocytosis, including endophilin, dap160, bchs (blue cheese), spict (spichthyin), and rab11 (Dickman

Received May 21, 2008; revised July 1, 2008; accepted July 11, 2008.

This work was supported by postdoctoral grants from the Damon Runyon Cancer Research Foundation and the Charles King Trust of the Medical Foundation (A.A.R.), and by grants from the National Institutes of Health (J.T.L.). We thank Hugo Bellen, Graeme Davis, Barry Ganetzky, Bruce Goode, Kate O'Connor-Giles, Jack Taunton, Defne Yarar, the Developmental Studies Hybridoma Bank, and the Drosophila Genomics Resource Center for reagents; Aline Blunk, Kate O'Connor-Giles, Bruce Goode, Sarah Huntwork-Rodriguez, Sudipta Saraswati, Robin Stevens, and Defne Yarar for helpful scientific discussions; and Aline Blunk, Danielle Pigneri, Betty Yang, and Rebecca Rodal for technical assistance.

Correspondence should be addressed to Avital A. Rodal, The Picower Institute for Learning and Memory, Departments of Biology and Brain and Cognitive Sciences, Massachusetts Institute of Technology, 46-3251, 43 Vassar Street, Cambridge, MA 02139. E-mail: arodal@mit.edu.

DOI:10.1523/JNEUROSCI.2304-08.2008

Copyright $\odot 2008$ Society for Neuroscience $\quad$ 0270-6474/08/288316-10\$15.00/0 et al., 2006; Khodosh et al., 2006; Wang et al., 2007), exhibit NMJ overgrowth, characterized by an excess of "satellite" boutons budding from axon terminal arbors. These proteins localize together with growth-regulatory molecules to a domain called the periactive zone, which surrounds sites of synaptic vesicle release (Roos and Kelly, 1999; Sone et al., 2000). It has been proposed that synaptic overgrowth in endocytosis mutants is attributable to a defect in the internalization and downregulation of signaling complexes driving synaptic growth (Dickman et al., 2006). One likely signal transduction cascade whose activity may be modulated after endocytosis functions downstream of the TGF- $\beta$ /bone morphogenic protein (BMP) family member Glass Bottom Boat (Gbb) (Marqués and Zhang, 2006; Collins and Diantonio, 2007; O'Connor-Giles et al., 2008). However, the specific steps of endocytosis in which receptor activation and downregulation occur have not been identified.

Actin polymerization generates forces at multiple stages of endocytosis (Kaksonen et al., 2006). Many endocytic accessory proteins tie actin polymerization to membrane traffic, but their precise functions are not understood. Rapid actin polymerization depends on nucleation promoting factors, including the actinrelated protein 2/3 (Arp2/3) complex. The Arp2/3 complex requires activation by the Wiskott-Aldrich syndrome protein (WASp) family of proteins, which contain an autoinhibitory and GTPase-binding domain, an $\mathrm{SH} 3$ domain-binding proline-rich domain, and an Arp2/3 complex-activating verprolin-centralacidic (VCA) domain. Ligands such as the small GTPase Cdc42 and $\mathrm{SH} 3$ domain proteins release WASp autoinhibitory interactions, leading to Arp2/3 complex activation (Stradal and Scita, 2006). 
One class of WASp ligands includes members of the FCH-BIN amphiphysin RVS (F-BAR)/SH3 family of proteins, which bind to dynamin and WASp via their SH3 domains, and induce lipid tubulation via their F-BAR domains, thus linking membrane invagination and actin polymerization (Itoh and De Camilli, 2006; Tsujita et al., 2006). Drosophila Nervous Wreck (Nwk) is a conserved F-BAR/SH3 domain protein. $n w k$ mutants exhibit synaptic overgrowth with excess satellite boutons reminiscent of endocytic mutants (Coyle et al., 2004), and Nwk interacts physically and genetically with Wsp, the single Drosophila WASp homolog (Ben-Yaacov et al., 2001; Coyle et al., 2004). Recent evidence shows that Nwk might directly link BMP-signaling complexes to the endocytic machinery (O'Connor-Giles et al., 2008).

Here, we demonstrate that Nwk binds to endocytic proteins and directly controls Wsp/Arp2/3 actin polymerization via specific SH3 domains. We show that Cdc42 cooperates with Nwk both in activating Wsp/Arp2/3 actin polymerization in vitro and in regulating synaptic growth in vivo. Cdc 42 and Wsp function in recycling endosomes (Parsons et al., 2005; Balklava et al., 2007), and we find that the recycling endosome marker Rab11 colocalizes with Nwk in periactive zones. Together, our results suggest that periactive zones are the synaptic equivalent of recycling endosomes, from which signals for synaptic growth are regulated by actin polymerization-dependent membrane traffic.

\section{Materials and Methods}

Fly stocks. Flies were cultured using standard media and techniques. Upstream activator sequence (UAS)-Nwk lines were constructed in the NWK cDNA by site-directed mutagenesis of pUAST-NWK (Coyle et al., 2004) and injected into $w^{1118}$ flies at the Duke Model Systems Transgenic Facility (Duke University, Durham, NC) or at Genetic Services (Cambridge, MA).

Immunohistochemistry and analysis of NMJ morphology. For analysis of NMJ morphology, flies were cultured at low density at $25^{\circ} \mathrm{C}$. Wild-type controls for each experiment were selected to be closely genetically matched to experimental genotypes. Wandering third-instar larvae were dissected in calcium-free HL3.1 saline (Feng et al., 2004), fixed for $30 \mathrm{~min}$ in HL3.1 containing 4\% formaldehyde, then washed in PBS and stained with anti-complexin (Cpx) (Huntwork and Littleton, 2007) and antiDiscs large (Dlg) (Parnas et al., 2001) antibodies. Larvae were imaged on a Zeiss Axioplan equipped with a Pascal laser-scanning head. NMJs on muscle 6/7, segment A3 and muscle 4, segments A2-A3 were selected for analysis. Both type $1 \mathrm{~b}$ and type $1 \mathrm{~s}$ boutons were quantified on muscle $6 / 7$. Only type Ib innervation, delineated by extensive postsynaptic anti-Dlg staining, was quantified on muscle 4 . Satellite boutons were defined as strings of five or fewer boutons extending from the main axis of the NMJ. Muscle surface area was visualized by anti-Dlg staining and measured using Pascal software. wit ${ }^{\mathrm{A} 12 / \mathrm{B} 11}$ and both $c d c 42 ; n w k$ animals and their matched $y, w$ wild-type controls have significantly smaller muscle area than $n w k$ or $w^{1118}$ larvae (supplemental Table S1, available at www.jneurosci.org as supplemental material), and bouton number is proportional to muscle area (Lnenicka and Keshishian, 2000), thus all NMJ quantifications were normalized to muscle area. Un-normalized data are also tabulated in supplemental Table S1, available at www. jneurosci.org as supplemental material. All errors shown are SEM. Statistical significance was calculated using unpaired Student's $t$ tests, with $p<0.05, p<0.01$, and $p<0.005$.

Protein purification. A fragment of Drosophila Wsp (Wsp143, lacking amino acids 1-143) was cloned into a pET-28a (EMD Biosciences)-based His-HA-tagged vector used previously to purify a similar fragment of mammalian N-WASp (Co et al., 2007). Escherichia coli strain BL21(DE3) expressing this construct was grown to $\log$ phase at $37^{\circ} \mathrm{C}$, then chilled to room temperature and induced with $0.4 \mathrm{~mm}$ isopropyl- $\beta$-Dthiogalactopyranoside for $4 \mathrm{~h}$. Cells were harvested and frozen at $-80^{\circ} \mathrm{C}$. Cell pellets were thawed in $20 \mathrm{~mm}$ sodium phosphate, $500 \mathrm{~mm} \mathrm{NaCl}, 20$ mм imidazole, $0.5 \mathrm{~mm}$ DTT, $0.5 \%$ (v/v) Triton X-100, $\mathrm{pH} 8.0$, supple- mented with $0.5 \mu \mathrm{g} / \mathrm{ml}$ pepstatin, leupeptin, and aprotonin and $1 \mathrm{~mm}$ PMSF, and loaded onto a $1 \mathrm{ml}$ HiTrap nickel column (GE Healthcare). Wsp143 was eluted in the same buffer with $300 \mathrm{~mm}$ imidazole, concentrated, and gel filtered on a Superose 12 HR 10/30 column equilibrated in 20 mм HEPES, $200 \mathrm{~mm} \mathrm{NaCl}, 0.5 \mathrm{~mm}$ DTT, pH 7.5. Peak fractions were flash frozen in liquid $\mathrm{N}_{2}$. Glutathione $S$-transferase (GST)-WASp-VCA was generated by cloning a fragment of Drosophila WASp (amino acids 427-527) into pGEX-4T-1 (Promega). GST-WASp-VCA was purified from bacterial cell lysates on glutathione agarose (GE Healthcare) and eluted with glutathione according to manufacturer instructions. Glutathione eluates were gel filtered as described above for Wsp143.

A fragment of Nwk (Nwk $\Delta \mathrm{C})$ encoding amino acids 1-754 was cloned into pTrcHisA (Invitrogen) and site-directed mutants were introduced to generate variants $\mathrm{SH} 3 \mathrm{a}^{*}(\mathrm{~W} 531 \mathrm{~A})$ and $\mathrm{SH} 3 \mathrm{~b}^{*}$ (W677A). E. coli strain BL21(DE3) expressing these constructs were grown as described above for Wsp143. Cell lysates were purified on $1 \mathrm{ml}$ HiTrap nickel columns (GE Healthcare) as described above. Peak fractions were diluted 10-fold in $20 \mathrm{~mm}$ Tris, $0.1 \mathrm{~mm}$ EDTA, $0.5 \mathrm{~mm}$ DTT, $\mathrm{pH}$ 8.5, and applied to a monoQ HR 5/5 anion exchange column (GE Healthcare). Proteins were eluted using a gradient of $0-600 \mathrm{~mm} \mathrm{NaCl}$, and peak fractions were concentrated and applied to a Superose 12 HR 10/30 gel filtration column (GE Healthcare) equilibrated in $20 \mathrm{~mm}$ Tris, $50 \mathrm{~mm} \mathrm{NaCl}, 0.1 \mathrm{~mm}$ EDTA, $0.5 \mathrm{~mm}$ DTT, $\mathrm{pH}$ 7.5. Peak fractions were pooled, flash frozen in liquid $\mathrm{N}_{2}$, and stored at $-80^{\circ} \mathrm{C}$.

Mammalian Cdc42 $\triangle$ CAAX was expressed, purified, and exchanged for guanylyl imidodiphosphate (GMP-PNP) as described previously (Abdul-Manan et al., 1999; Higgs and Pollard, 2000). Drosophila GSTCdc42(Q61L) (Bouslama-Oueghlani et al., 2007) was purified on glutathione agarose and eluted with glutathione using standard procedures, and applied to a Superose 12 HR 10/30 gel filtration column equilibrated in $20 \mathrm{~mm}$ Tris, $150 \mathrm{~mm} \mathrm{NaCl}, 2 \mathrm{~mm} \mathrm{MgCl}_{2}, 2 \mathrm{~mm}$ DTT, pH 7.5. Peak fractions were supplemented with $5 \%(\mathrm{v} / \mathrm{v})$ glycerol, frozen in liquid $\mathrm{N}_{2}$, and stored at $-80^{\circ} \mathrm{C}$.

Bovine Arp2/3 complex and pyrene-labeled rabbit muscle actin were purchased from Cytoskeleton. Unlabeled rabbit muscle actin was purified from acetone powder and freshly gel filtered as described previously (Moseley et al., 2006).

Antibodies. A fragment of Drosophila WASp encoding amino acids 1-179 was cloned into pTrcHisA (Invitrogen). Cell lysates were purified on a $1 \mathrm{ml}$ HiTrap nickel column as above for Nwk $\Delta$ C. Peak fractions from this column were concentrated, applied to a Superose 12 HR 10/30 gel filtration column equilibrated in PBS, and injected into chickens (Aves Labs). The IgY fraction from immunized chicken eggs was purified against the antigen immobilized on nitrocellulose as described previously (Roos and Kelly, 1998). A fragment of Nwk encoding amino acids 630754 and encompassing SH3b was used to immunize rabbits (Invitrogen) to generate $\alpha$-Nwk antibody \#69009, which was used for immunoblots (see Fig. 4A). $\alpha$-Nwk antibody \#970 (Coyle et al., 2004) was used for immunoblots and immunohistochemistry (see Figs. 4, 8). $\alpha$-Dynamin, $\alpha$-Dap160 (Roos and Kelly, 1998), and $\alpha$-Rab11 (Khodosh et al., 2006) antibodies have been described previously.

GST pull-downs, immunoprecipitations, and yeast two-hybrid assays. For GST pull-downs, a fragment of Nwk encoding amino acids 1-754 or SH3 domain variants W531A and W677A was cloned into pGEX4T-1 (Promega). GST-Dap160-SH3 was generated by cloning a fragment of Dap160-RA encoding amino acids 638-end into pGEX-5X (Promega). GST fusions were purified from bacterial lysates on glutathione agarose in PBS with 0.5 mM DTT, and beads were aliquoted and flash frozen in liquid $\mathrm{N}_{2}$. Protein concentrations were normalized using empty beads. Lysates were made from fly heads in $20 \mathrm{~mm}$ HEPES, pH 7.5, $50 \mathrm{~mm} \mathrm{KCl}$, $0.5 \mathrm{~mm}$ EGTA, $1 \mathrm{~mm} \mathrm{MgCl}_{2}$, and $0.2 \%$ (v/v) Igepal CA630, supplemented with protease inhibitors as described above, using a Teflon homogenizer. $n w k^{2}$ heads were used to eliminate competition for binding to and dimerization with endogenous Nwk. Lysates were applied to beads for $3 \mathrm{~h}$ at $4^{\circ} \mathrm{C}$. For immunoprecipitations, lysates from wild-type $\left(w^{1118}\right)$ and $n w k^{2}$ heads were incubated for $2 \mathrm{~h}$ with anti-dynamin antibodies (Roos and Kelly, 1998), then for $1 \mathrm{~h}$ with protein G Sepharose (GE Healthcare). Beads were then pelleted and washed three times with $1 \mathrm{ml}$ of lysis buffer, resuspended in 1/4 original lysate volume of denaturing sample buffer, 
and processed for immunoblotting and detection using a LICOR Odyssey infrared scanner (LICOR).

Yeast two-hybrid assays were conducted using the Matchmaker system (Clontech). Nwk bait constructs were cloned into pGBKT7 and tested for interaction with Dap160 eps15 homology (EH) (amino acids 1-287) and Dap160 $\Delta$ EH (amino acids 259-end) cloned into pAct2 by selecting for growth on minimal yeast media lacking leucine, tryptophan, and histidine, and containing $10 \mathrm{~mm} 3$-aminotriazole. Yeast colonies were scored for growth after $2 \mathrm{~d}$ relative to empty bait plasmid with Dap160AEH or Dap160EH prey and empty prey plasmid with Nwk bait.

Actin polymerization kinetics. Rabbit muscle actin (5\% pyrene labeled) was prepared, exchanged from $\mathrm{Ca}^{2+}$ to $\mathrm{Mg}^{2+}$, and assembled as described previously (Moseley et al., 2006). Assembly was monitored on a spectrofluorometer (Photon Technology International) or a microplate reader (TECAN), using an excitation wavelength of $365 \mathrm{~nm}$ and an emission wavelength of $405 \mathrm{~nm}$. Rates were calculated from slopes of curves in the linear range, and concentration curves were calculated using GraphPad Prism software (GraphPad Software).

\section{Results}

Synaptic overgrowth in $n w k$ requires the Gbb cascade

Nwk is required to constrain synaptic growth at the Drosophila larval NMJ (Coyle et al., 2004), and members of the Nwk family regulate actin assembly tied to endocytosis, consistent with evidence that endocytosis may mediate downregulation of signals for synaptic growth (Wang et al., 2007). The TGF- $\beta$ /BMP family member Gbb regulates synaptic growth through its receptors Wishful Thinking (Wit), Thickveins (Tkv), and Sax (Saxophone), which are expressed in motor neurons (Marqués and Zhang, 2006). We tested whether the activity of this signaling cascade was required for the synaptic overgrowth phenotype of $n w k$ mutants, which is characterized by an increase in overall bouton number and satellite bouton formation. Mutations in Wit cause reduced synaptic growth at the NMJ (Marqués and Zhang, 2006). The synaptic overgrowth phenotype of $n w k$ mutants was completely suppressed by additional mutation of wit, and wit ${ }^{A 12 / B 11}, n w k^{1}$ double mutants were not significantly different from wit ${ }^{A 12 / B 11} \mathrm{mu}-$ tants (Fig. 1, supplemental Table 1a-c, available at www.jneurosci.org as supplemental material), indicating that the Gbb cascade is required for synaptic overgrowth in $n w k$. Similar results were obtained with $g b b^{1 / 2} ; n w k^{2}$ double mutants (data not shown). Thus, Nwk constrains synaptic growth by acting upstream of synaptic growth signal output rather than by directly affecting the machinery that drives morphological changes in synaptic boutons.

\section{Nwk SH3 domains interact with the endocytic machinery}

We tested whether Nwk might regulate synaptic growth signals through interactions with the endocytic machinery. Members of the F-BAR/SH3 family of proteins function in endocytosis and bind to proline-rich regions in dynamin and WASp via their $\mathrm{SH} 3$ domains (Itoh et al., 2005; Tsujita et al., 2006), and Nwk requires Dap160, the Drosophila homolog of the endocytic protein inter- sectin 1s, for proper localization to periactive zones (Koh et al. 2004). GST-Nwk $\Delta$ C immobilized on beads efficiently precipitated Dynamin, Dap160, and Wsp (Fig. 2A) from Drosophila head extracts. We generated point mutations in each Nwk SH3 domain [W531A $\left(\mathrm{SH}_{3} \mathrm{a}^{*}\right)$ and W677A $\left(\mathrm{SH} 3 \mathrm{~b}^{*}\right)$ ] that are predicted to abolish binding to proline-rich targets (Fazi et al., 2002). GST pull-downs with these mutant proteins revealed that $\mathrm{SH} 3 \mathrm{a}$ is required for Nwk-dynamin and Nwk-Wsp interactions and that SH3b is required for Nwk-Dap160 interactions (Fig. 2A).

To determine whether Nwk SH3a interactions with Wsp and dynamin are mutually exclusive, we immunoprecipitated endogenous dynamin complexes from wild-type and $n w k$ mutant Drosophila head extracts. Dynamin antibodies efficiently precipitated Nwk and Dap160, but not Wsp (Fig. 2 B), indicating that Nwk is predominantly associated with dynamin and that these dynaminNwk complexes lack Wsp. Thus, Nwk may switch interactions between Wsp and dynamin. Although Nwk binds to both Dap160 and to dynamin, dynamin-Dap160 interactions did not require Nwk (Fig. 2 B).

Dap160 contains only one predicted SH3-binding site, which is located between its two $\mathrm{EH}$ domains. However, this region (amino acids 1-287) was not sufficient for interactions between Dap160 and Nwk in the yeast two-hybrid assay. In contrast, the C terminus of Dap160 (amino acids 259-end) interacted with Nwk in a manner that depended on Nwk-SH3b in the yeast two-hybrid assay (Fig. 2C), recapitulating the results from GST pull-downs. Thus, Dap160/Nwk-SH3b interactions do not depend on the single predicted SH3-binding site in Dap160 but on another determinant in the $\mathrm{C}$ terminus. We tested whether the purified 
A
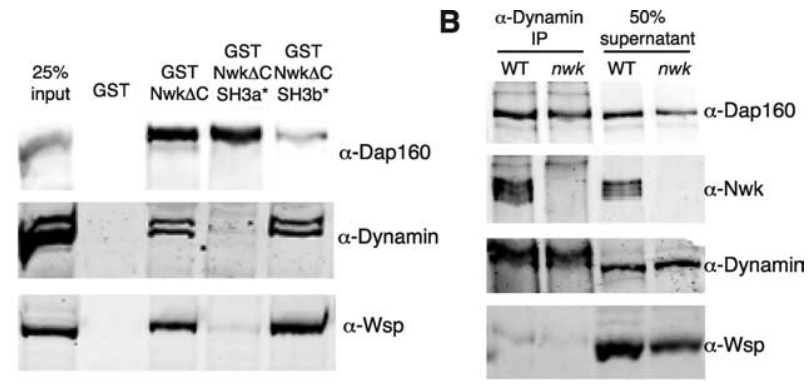

C

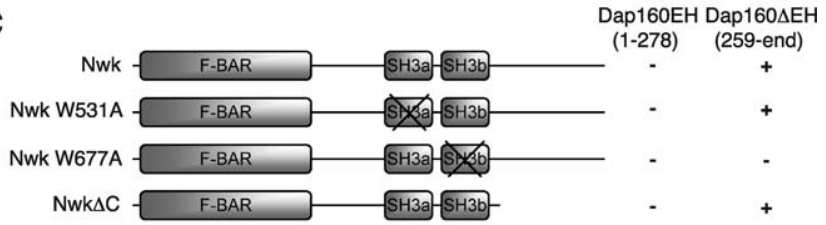

D

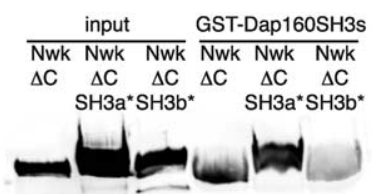

$\mathbf{E}$

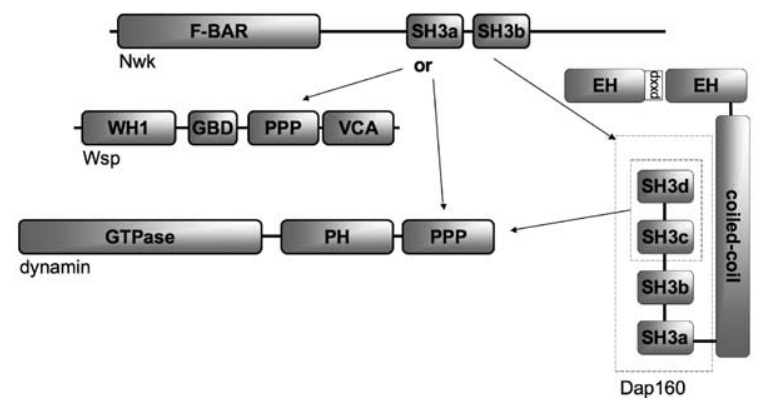

Figure 2. Ligands of Nwk SH3 domains $A$, Equal amounts of GST fusion proteins bound to glutathione agarose were used to precipitate binding partners from $n w k^{2}$ Drosophila head extracts. Equal amounts of precipitates were immunoblotted with the indicated antibodies. $\boldsymbol{B}$, Anti-dynamin antibodies were used to precipitate dynamin-containing protein complexes from wild-type and $n w k^{2}$ Drosophila head extracts. Equal amounts of precipitates were immunoblotted with the indicated antibodies. C, Summary of yeast two-hybrid assay results for interaction of Nwk and Nwk $\Delta C$ (amino acids 1-754) with Dap160EH (amino acids 1-287) and Dap160 $\Delta$ EH (amino acids 259 - end). D, GST-Dap160SH3 (amino acids 638 - end) was used to precipitate purified 6-His-tagged Nwk $\Delta \mathrm{C}$ and $\mathrm{SH} 3$ domain variants. Equal amounts of precipitates were immunoblotted with $\alpha$-Nwk antibody \#97009. Only background reactivity of GST-Dap160SH3 (that is the same molecular weight as Nwk $\Delta C$ ) is visible in Nwk $\Delta C$-SH3b* reactions. $\boldsymbol{E}$, Model of interactions between Nwk, Dap160, dynamin, and Wsp. The interaction of Dap160 SH3a-SH3b with dynamin was demonstrated previously (Roos and Kelly, 1998).

C-terminal region of Dap160 (Dap160-SH3, amino acids 638end, containing four $\mathrm{SH} 3$ domains) could bind to purified Nwk $\Delta$ C. Dap160-SH3 bound specifically to Nwk $\Delta \mathrm{C}$ and Nwk $\Delta$ C-SH3a ${ }^{*}$, but not Nwk $\Delta$ C-SH3b* (Fig. $2 D$ ), recapitulating the results from extracts (Fig. $2 A$ ). Because these binding reactions contained only purified proteins, and no predicted SH3-binding site exists in the Dap160 C terminus, we hypothesize that either Nwk-SH3b binds directly to a noncanonical SH3binding site in this region of Dap160 (Duke-Cohan et al., 2006), or that Nwk-SH3b is required indirectly via intramolecular interactions within Nwk to reveal a binding site for Dap160 SH3 domains. In summary, our results show that Nwk interacts with components of the endocytic machinery via its $\mathrm{SH} 3$ domains (Fig. 2E), tying its function to membrane traffic.
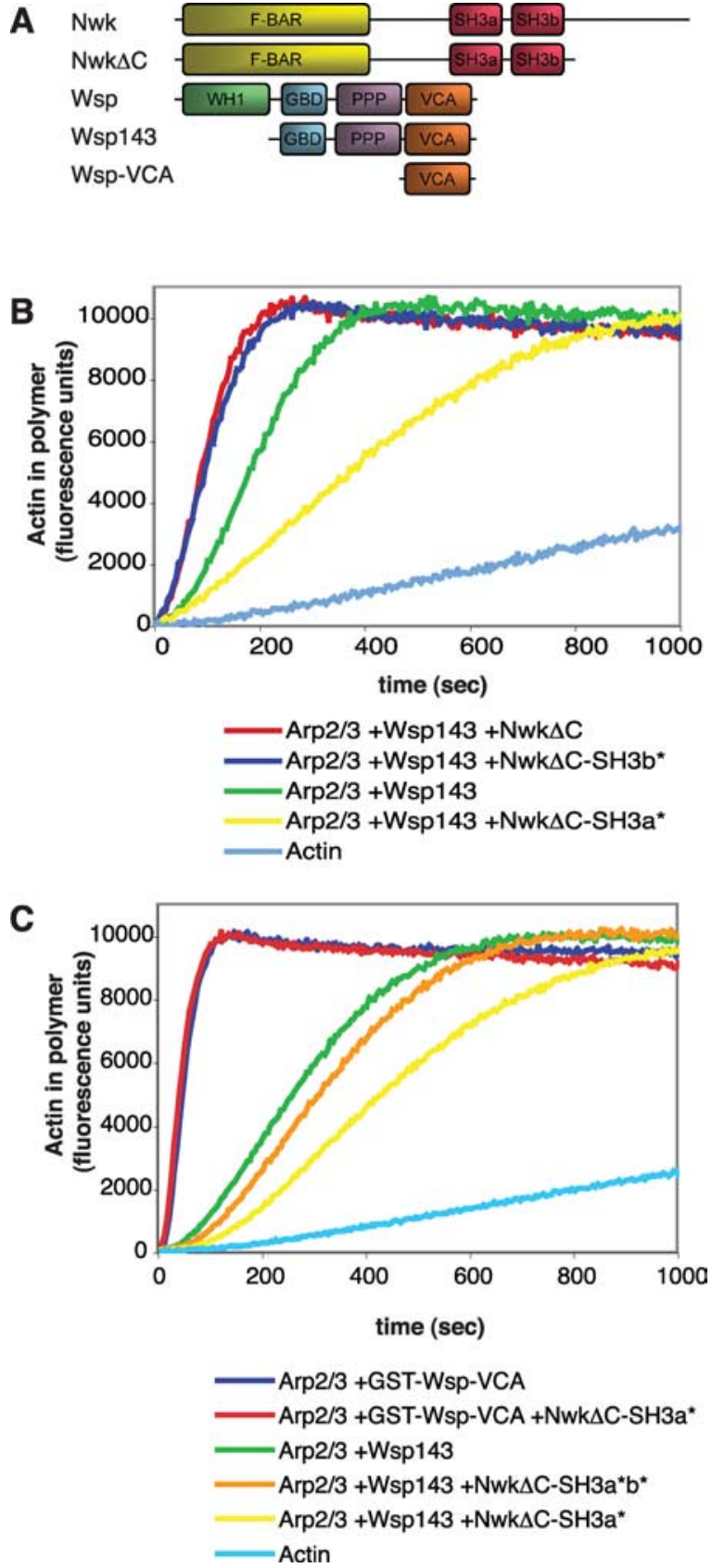

Figure 3. Function of NwkSH3 domains in activation of Wsp/Arp2/3 actin polymerization. $A$, Schematic of protein fragments used for actin polymerization kinetics. B, C, $2.5 \mu \mathrm{m}$ actin $(5 \%$ pyrene-labeled) was assembled in the presence of $20 \mathrm{~nm}$ Arp2/3 complex, $40 \mathrm{~nm}$ Wsp143 or 40 nм GST-WspVCA, and $1 \mu \mathrm{m}$ Nwk $\Delta C$. Nwk-SH3a mediates release of Wsp autoinhibition, whereas Nwk-SH3b plays an inhibitory role.

Nwk activates WASp-Arp2/3-mediated actin polymerization Nwk interacts with Wsp (Fig. 2), which activates Arp2/3 complex-mediated actin polymerization to generate force for endocytic events (Kaksonen et al., 2006). To determine the effects of Nwk-Wsp interactions on the actin-nucleation activity of the Arp $2 / 3$ complex, we tested the effects of purified Nwk, Wsp, and Arp $2 / 3$ complex on the polymerization of actin. We purified a fragment of Wsp (Wsp143) (Fig. 3A), which contains the Cdc42binding, proline-rich, and Arp2/3-activating VCA domains of Wsp, retaining autoinhibitory interactions between its $\mathrm{N}$ and $\mathrm{C}$ termini (Co et al., 2007). Nwk $\Delta$ C (Fig. 3A) (lacking the region $\mathrm{C}$ terminal to the $\mathrm{SH} 3$ domains), which rescues $n w k$ phenotypes in 
vivo (see below), stimulated the activity of Wsp143 (Fig. 3B) but had no effect on actin polymerization on its own or with Arp2/3 alone (data not shown). We next tested the role of each Nwk SH3 domain in activation of Wsp. Nwk $\Delta \mathrm{C}-\mathrm{SH} 3 \mathrm{~b}^{*}$ activated Wsp143 identically to wild-type Nwk $\Delta$ C, consistent with biochemical interactions showing that Nwk-SH3a mediates Wsp-Nwk associations. Nwk $\Delta \mathrm{C}$ $\mathrm{SH}_{3} \mathrm{a}^{\star}$ was unable to stimulate Wsp143/ Arp $2 / 3$ and in fact uncovered an inhibitory effect for Nwk on Wsp/Arp2/3 complex. Nwk $\Delta$ C-SH $3 \mathrm{a}^{*}$ had no inhibitory effect on Wsp-VCA domain (nonautoinhibited) activation of the Arp2/3 complex, suggesting that inhibition was mediated through the proline-rich region of Wsp143 (Fig. 3C). The inhibitory effect of Nwk $\Delta \mathrm{C}-\mathrm{SH} 3 \mathrm{a}^{*}$ was mainly relieved by additionally mutating Nwk-SH3b (Fig. 3C), suggesting that the $\mathrm{SH} 3 \mathrm{~b}$ domain mediates the negative regulatory function and that ligands of SH3b could relieve negative regulation of Nwk-Wsp activation. These results are consistent with $\mathrm{SH} 3 \mathrm{~b}$ acting directly on the proline-rich region of Wsp because our actin polymerization assays contain only purified components, although $\mathrm{SH} 3 \mathrm{~b}$ is not sufficient for precipitation of Wsp from Drosophila extracts (Fig. 2A). This may reflect a low affinity interaction between $\mathrm{SH} 3 \mathrm{~b}$ and Wsp, or competing SH3b or Wsp proline-rich domain ligands in extracts. Wsp contains one predicted Nwk SH3abinding site and three predicted Nwk SH3b-binding sites (Ferraro et al., 2007). One SH3b-binding site overlaps with the SH3a binding site, suggesting that $\mathrm{SH} 3 \mathrm{~b}$ could prevent activation of Wsp by reducing conformational change in the specific region of Wsp that is required for activation through SH3a. In summary, our biochemical analysis indicates that Nwk-SH3a directly activates Wsp-dependent actin polymerization and that Nwk-SH3b ligands may regulate this activation process.

\section{Nwk function in vivo depends on $\mathrm{SH} 3$ domain interactions}

To test the role of Nwk SH3 domains on its in vivo function, we expressed full-length Nwk transgenes containing SH3 point mutants in a $n w k$ null background (Fig. $4 A$ ). Overexpression of wildtype Nwk does not affect synaptic growth (see below). Nwk mutant transgenes localized to synaptic boutons in a similar pattern ["periactive zones," surrounding the active zone marker Bruchpilot (Brp)] to wild-type Nwk (Fig. 4B), although the Nwk$\mathrm{SH} 3 \mathrm{a}^{\star} \mathrm{b}^{*}$ double mutant appeared somewhat more diffuse. The predominant phenotype of $n w k$ mutants is an increase in overall bouton number. We quantified this phenotype in transgenic rescue animals and found that Nwk-SH3a* and Nwk-SH3b ${ }^{*}$ failed to restore normal synaptic growth, whereas a transgene containing a deletion of the Nwk $C$ terminus rescued synaptic overgrowth as well as full-length Nwk (Fig. $5 A, B$, supplemental Table $1 \mathrm{~d}$, available at www.jneurosci.org as supplemental material). Interestingly, the Nwk-SH3a* $\mathrm{b}^{\star}$ double mutant exhibited more severe synaptic overgrowth than the $n w k$ null mutant or the $\mathrm{SH} 3$ domain single mutants, suggesting that the $\mathrm{SH} 3$ domains have overlapping functions and that the Nwk-SH3a* $\mathrm{b}^{\star}$ double mutant elav ${ }^{\mathrm{C} 155} /+$ or $\mathrm{Y} ; \mathrm{UAS} /+; n w k^{1} / n w k^{2}$

NWK NWK NWK

NWK NWK

$S H 3 b^{*} \Delta C$
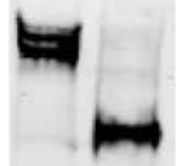

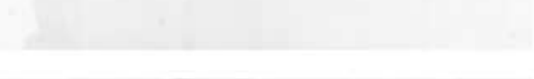
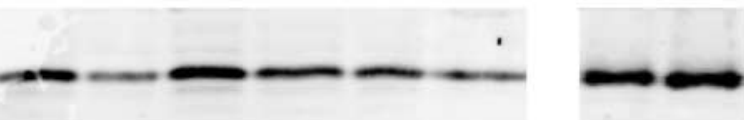

WK

NWK-SH3b* NWK-SH3a*b*

\section{.} $+$

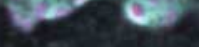
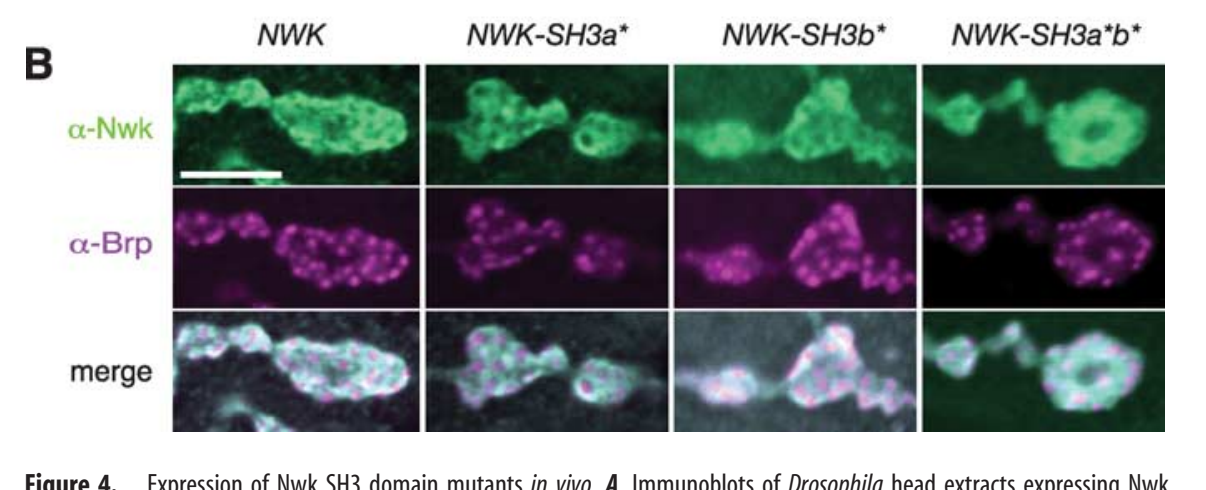

Figure 4. Expression of Nwk SH3 domain mutants in vivo. A, Immunoblots of Drosophila head extracts expressing Nwk sgenes. Cpx serves as a loading control. Left panels were immunoblotted with anti-Nwk antibody \#970 which was raised which was raised against Nwk-SH3b. B, Localization of Nwk mutant transgenes in synaptic boutons. Nwk periactive zone staining

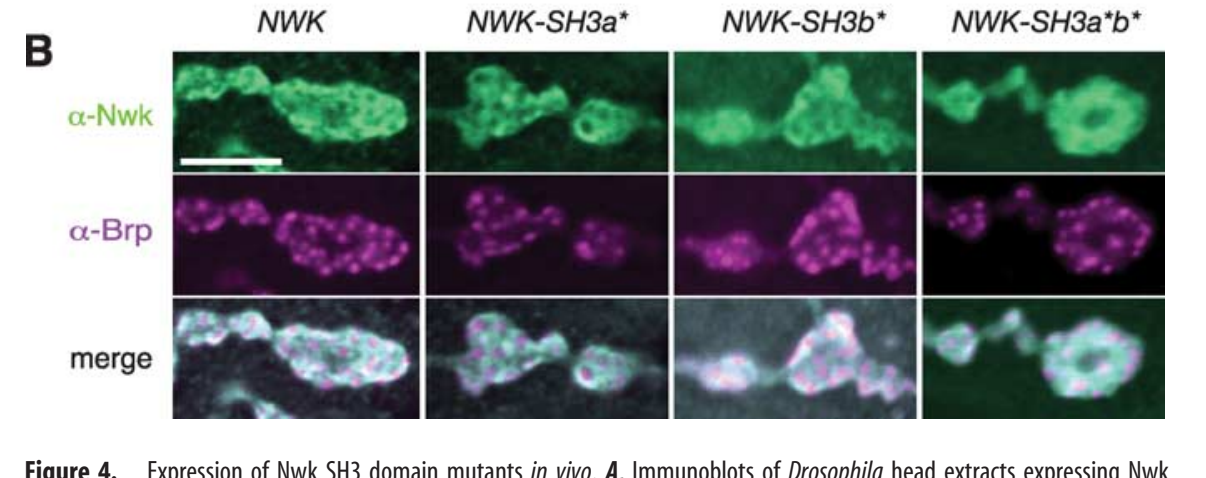

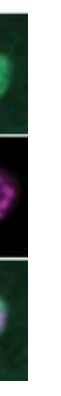


A
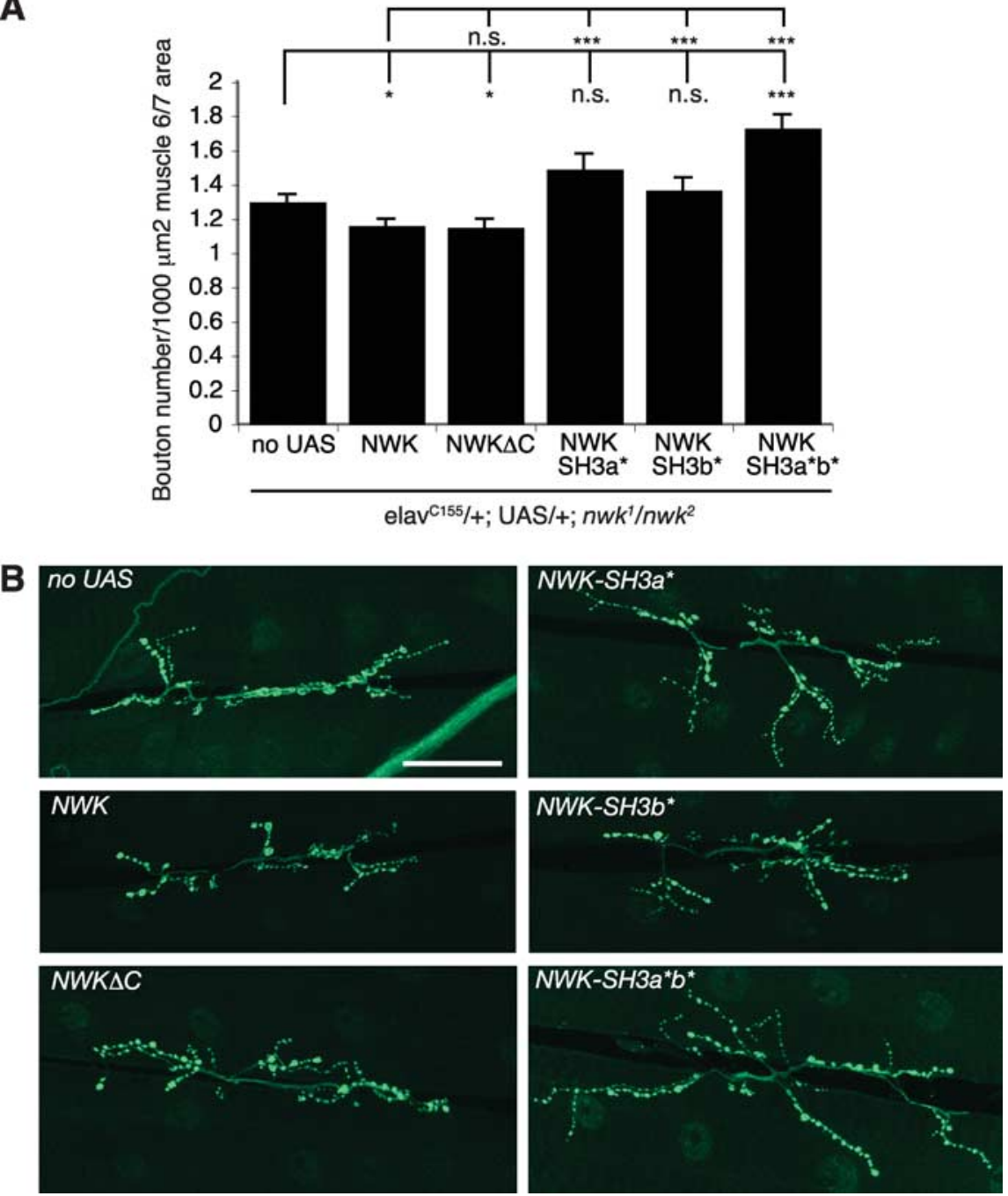

Figure 5. Rescue of $n w k$ mutant phenotype by mutant NWK transgenes. $\boldsymbol{A}$, Quantification of mean bouton number for segment A3, muscle 6/7. ${ }^{*} p<0.05,{ }^{* * *} p<0.005$. B, Representative confocal projections of anti-Cpx staining from NMJs quantified in $\boldsymbol{A}$. Scale bar, $50 \mu \mathrm{m}$. Raw data are tabulated in supplemental Table S1, available at www.jneurosci.org as supplemental material.

Cdc42 and Nwk may converge and stimulate Wsp together in vivo.

We analyzed the localization of Cdc42 at the Drosophila larval NMJ. Myc-tagged Cdc42, which complements the shared function of Nwk and Cdc42 (see below), localized to small presynaptic and postsynaptic puncta (Fig. $8 \mathrm{~A}$ ). Nwk localizes presynaptically at the NMJ in a honeycomb pattern that surrounds active zones (Coyle et al., 2004). Other proteins sharing this localization function in endocytosis and synaptic growth (Roos and Kelly, 1999; Sone et al., 2000). The presynaptic component of Cdc42 overlapped with Nwk localization in periactive zones (Fig. 8A). Recent work in Caenorhabditis elegans and mammalian cell culture indicates that $\mathrm{Cdc} 42$ functions in intracellular endosomal membrane traffic (Parsons et al., 2005; Balklava et al., 2007). Rab11 functions in recycling endosomes and rab11 mutants exhibit a large increase in satellite boutons at the Drosophila NMJ (Khodosh et al., 2006). Therefore, Nwk and Cdc42 may regulate synaptic growth at the actin-membrane interface of recycling endosomes. Rab11 localized in a very similar pattern to Cdc42, although it was difficult to conclusively determine the extent of colocalization of Cdc42 with Rab11 at the level of light micros- copy because of the small size and abundance of the puncta. However, Rab11 puncta exhibited a striking colocalization with Nwk in periactive zones (Fig. 8B). These results suggest that Nwk functions in a Rab11-positive compartment, in which Cdc42 has previously been shown to act, and that the periactive zone may be the synaptic equivalent of the recycling endosome.

Nwk interacts with components of the endocytic machinery, but the synaptic growth phenotype of $n w k$ mutants are not as severe as that of endocytic mutants such as shibire (shi)/dynamin and dap160 (Dickman et al., 2006). We hypothesized that in $n w k$ mutants, Cdc42 activation of Wsp may partially compensate for the absence of Nwk. The function of Cdc42 at the Drosophila NMJ has not been previously characterized. Dominant-negative and constitutively active alleles of Cdc42 cause axon targeting defects (Luo et al., 1994), complicating analysis of their effects on NMJ growth. Therefore, we analyzed lossof-function alleles of $C D C 42$, which do not exhibit targeting defects (Genova et al., 2000). Unlike more severe alleles of CDC42, the mild hypomorphic allele $c d c 42^{2}$ gives rise to viable third instar larvae (Fehon et al., 1997). To test whether Cdc42 and Nwk have overlapping roles at the larval NMJ, we generated $c d c 42^{2}$; $n w k$ double mutants. $c d c 42^{2}$ exhibited a significant increase in bouton number and satellite bouton number relative to wild-type controls, and $c d c 42^{2}$; nwk double mutants showed an additive effect on synaptic growth with double the number of satellite boutons compared with each single mutant (Fig. $8 C-E$, supplemental Table $1 \mathrm{f}-\mathrm{h}$, available at www.jneurosci.org as supplemental material). This phenotype was recapitulated using an independent null allele of $n w k$ and rescued by an myc epitope-tagged $C D C 42$ transgene, confirming that the effects were specific to $\mathrm{Cdc} 42$ and Nwk (Fig. 8C-E, supplemental Table S1f-h, available at www. jneurosci.org as supplemental material). Because $c d c 42^{2}$ is an extremely mild hypomorphic allele, these results are likely to underestimate the actual contribution of Cdc42 to synaptic growth. We conclude that Cdc42 and Nwk cooperate in vitro to control actin polymerization by Wsp and in vivo to regulate synaptic growth, suggesting that converging signals from $\mathrm{SH} 3$ domains and Cdc42 control Wsp activity at the synapse.

\section{Discussion}

Our findings demonstrate that the conserved F-BAR/SH3 protein Nwk interacts with the endocytic machinery and activates Wsp/Arp2/3 actin polymerization together with Cdc42 to regulate synaptic growth upstream of growth factor signaling. Mapping these interactions and activities provides a critical framework for determining the mechanism by which endocytic accessory proteins and the cytoskeleton control membrane deformation during endocytosis. 
A
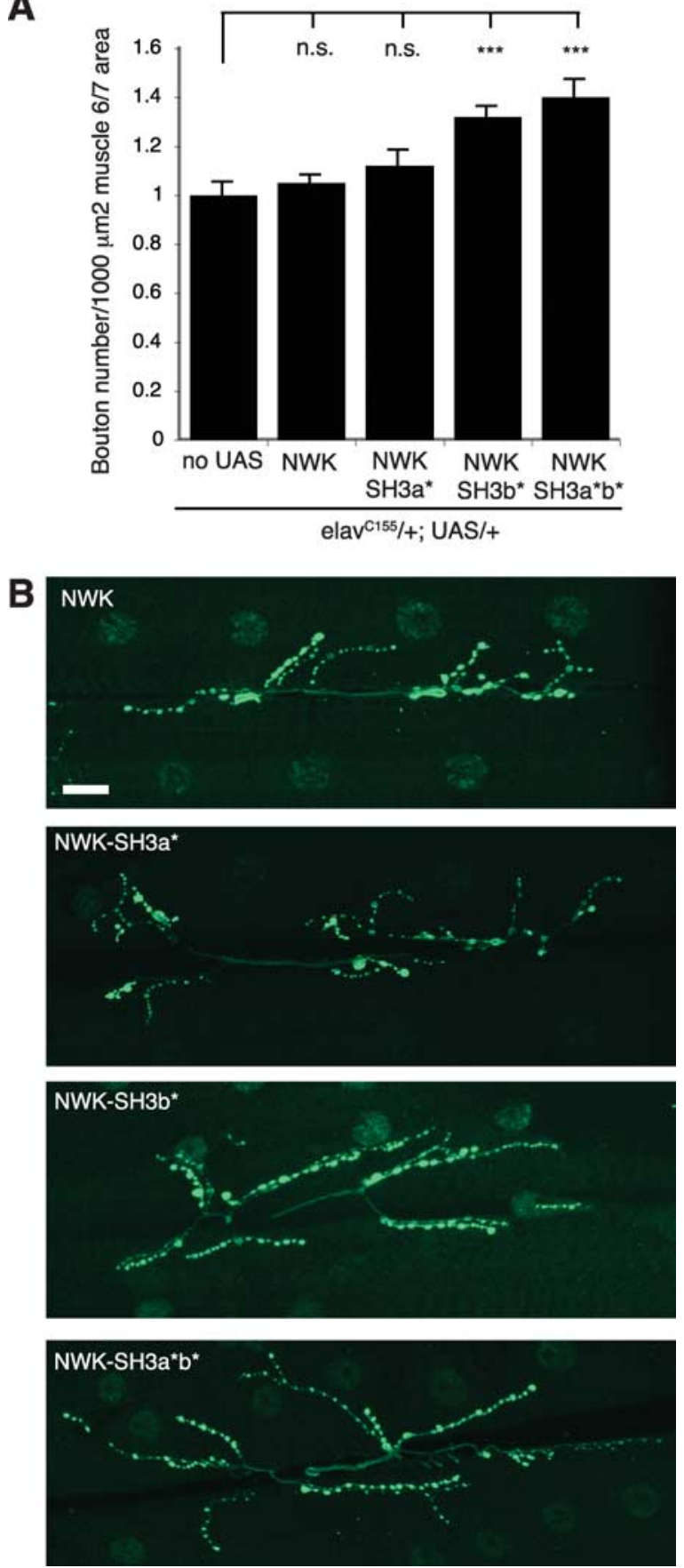

Figure 6. Dominant phenotypes of mutant $N W K$ transgenes. $A$, Quantification of mean bouton number for segment $A 3$, muscle $6 / 7 .{ }^{* *} p<0.005 . B$, Representative confocal projections of anti-Cpx staining from NMJs quantified in $\boldsymbol{A}$. Scale bar, $20 \mu \mathrm{m}$. Raw data are tabulated in supplemental Table S1, available at www.jneurosci.org as supplemental material.

Nwk activates Wsp/Arp2/3 actin polymerization via its $\mathrm{SH} 3 \mathrm{a}$ domain, and Nwk-SH3b is not required for Wsp binding or activation, but is required for the residual Wsp-inhibitory activity of Nwk when SH3a function is abolished. This activity may be more pronounced on endogenous Wsp, which is more tightly autoinhibited than recombinant WASp (Ho et al., 2004), raising the possibility that Nwk-SH3b could potently regulate Nwk$\mathrm{SH} 3 \mathrm{a}$-dependent activation of Wsp. Thus, ligands of Nwk-SH3b are in a position to serve as activators of Nwk and Wsp/Arp2/3 actin polymerization. Nwk-SH3b is required for interactions be-

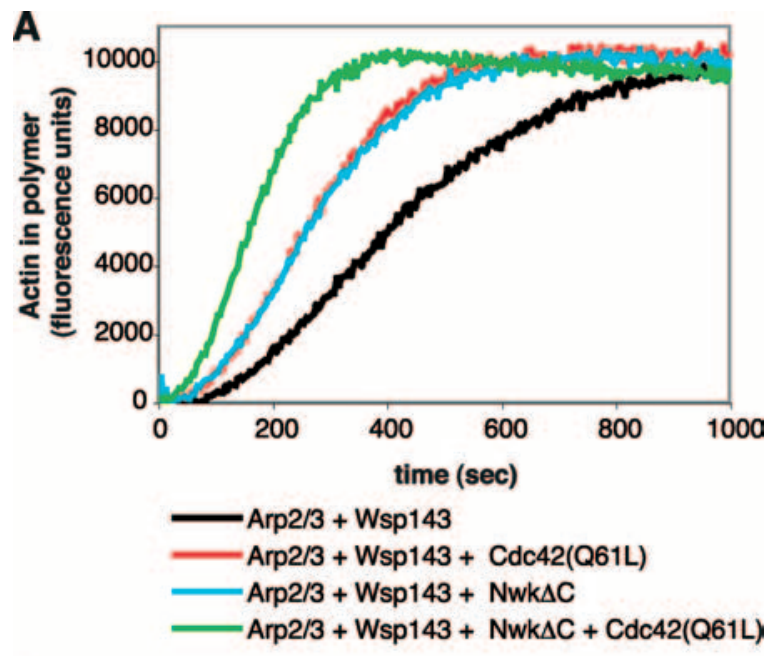

B

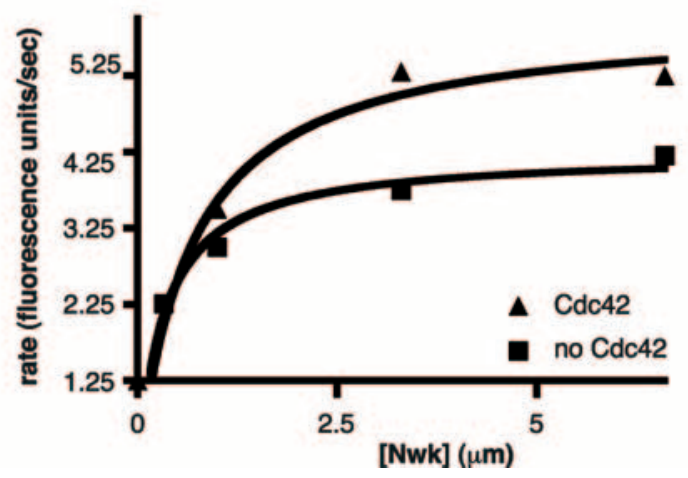

Figure 7. Cooperation of Cdc42 and Nwk in Wsp/Arp2/3 actin polymerization. A, $2.5 \mu \mathrm{m}$ actin (5\% pyrene-labeled) was assembled in the presence of $10 \mathrm{~nm}$ Arp2/3 complex, $17 \mathrm{nM}$ Wsp143, $1 \mu \mathrm{m} \mathrm{Nwk} \Delta C$, and $33 \mathrm{~nm}$ Cdc42(061L) (activated (dc42). B, Quantification of the effects of $\mathrm{Cd}(42$ and Nwk on the rate of actin assembly. $2.5 \mu \mathrm{m}$ actin (5\% pyrene-labeled) was assembled in the presence of $20 \mathrm{~nm}$ Arp2/3 complex, $17 \mathrm{~nm}$ Wsp143, $100 \mathrm{~nm}$ Cdc42 $\Delta$ CAAX-GMPPNP, and the indicated concentrations of Nwk $\Delta C$.

tween Nwk and Dap160, which is an excellent candidate for acting upstream of Nwk, because dap160 mutants exhibit synaptic overgrowth and temperature-sensitive seizures like those of $n w k$ mutants, and Nwk is mislocalized in dap160 NMJs (Koh et al., 2004; Marie et al., 2004). Recently, it was reported that the fragment of Dap 160 containing its last two SH3 domains is required for interaction with full-length Nwk in Drosophila extracts, leading to the hypothesis that the $\mathrm{C}$ terminal proline-rich region of Nwk mediates these interactions (O'Connor-Giles et al., 2008). Our results show instead that interactions between purified Nwk $\Delta$ C (i.e., Nwk lacking the C terminus) and both endogenous full-length Dap160 as well as purified Dap160 SH3 domaincontaining fragment depend on Nwk SH3b. Two possible interpretations can reconcile these results. Nwk SH3b may interact with a noncanonical SH3 domain-binding site in the intervening sequences between the Dap160 SH3 domains (Duke-Cohan et al., 2006). Alternatively, Nwk SH3b may function in an intramolecular interaction within Nwk that is required to expose one of several proline-rich sequences in the $\mathrm{N}$-terminal region Nwk for interaction with Dap160 SH3 domains. Thus, we conclude that Nwk SH3b is important for Dap160-Nwk interactions via an indirect or noncanonical mechanism. Further experiments will be needed to identify the Nwk-binding site on Dap160 and to confirm activity of Dap160 on Nwk in vitro.

Nwk-SH3a is required for interactions of Nwk with both dy- 

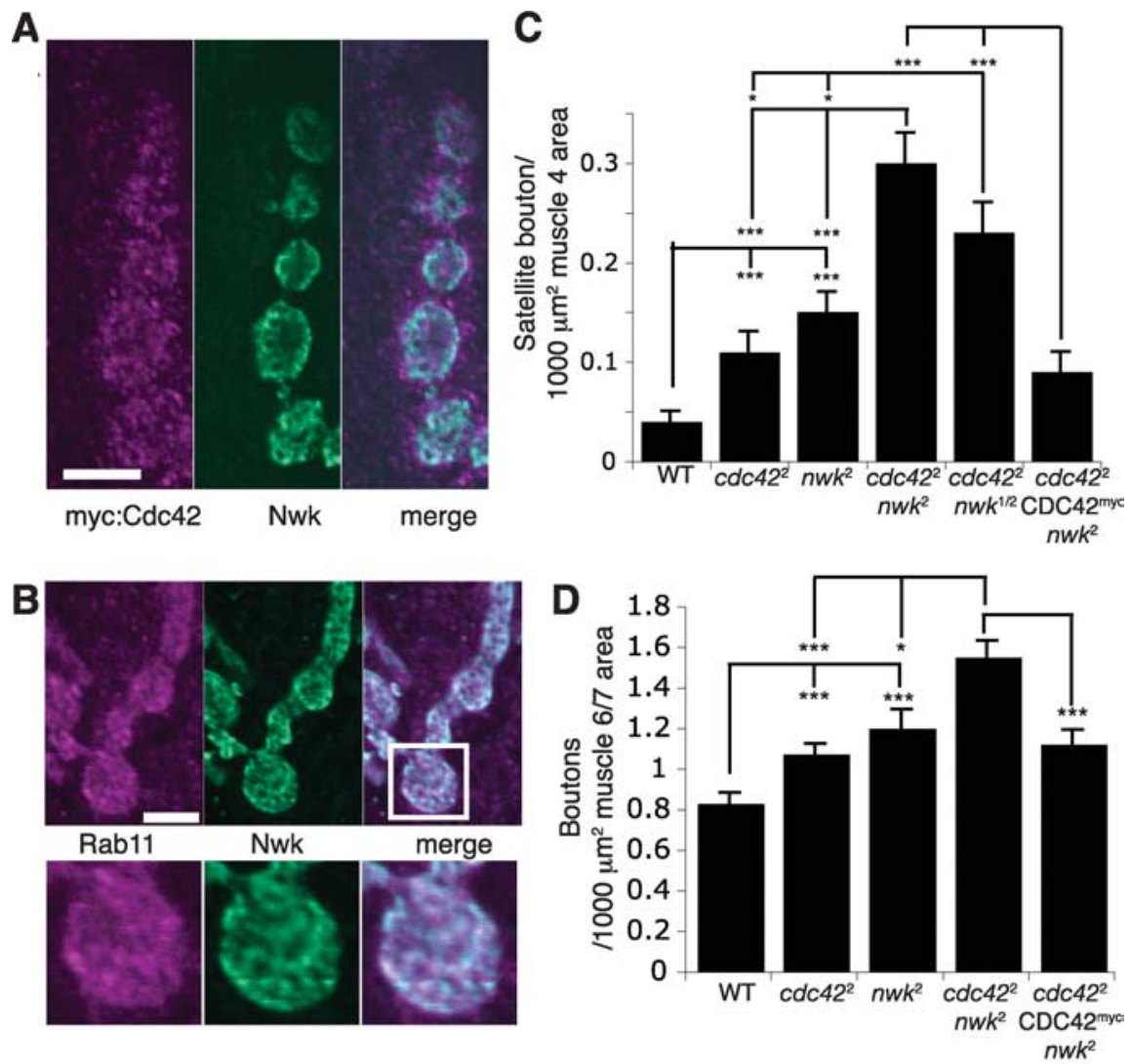

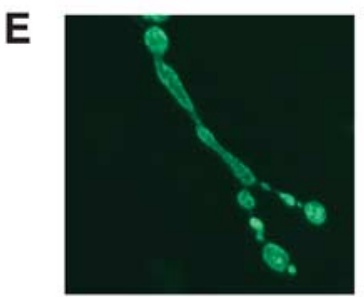

wild-type

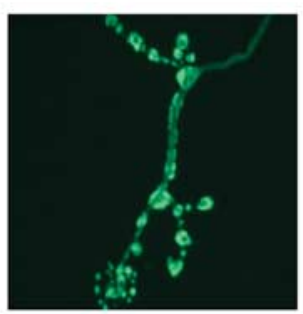

$c d c 42^{2} ; n w k^{2}$

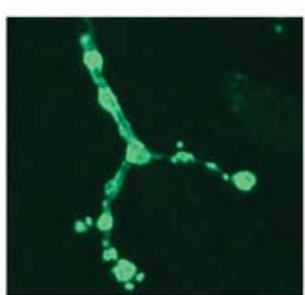

$\operatorname{cdc} 42^{2}$

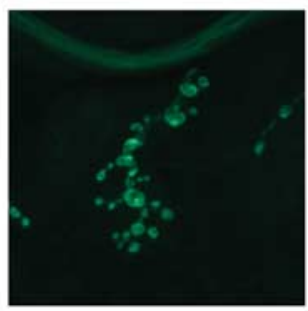

$c d c 42^{2} ; n w k^{1}$

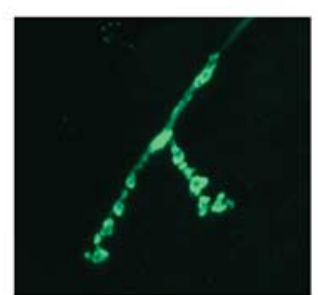

$n w k^{2}$

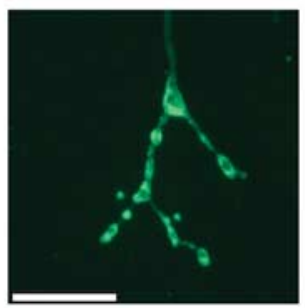

$c d c 42^{2} ; \operatorname{CDC} 42^{\text {myc }} ; n w k^{2}$

Figure 8. Cooperation of Nwk and Cdc42 in synaptic growth. $\boldsymbol{A}, \boldsymbol{B}$, Localization of $\mathrm{Cdc42} \mathrm{(A)} \mathrm{and} \mathrm{Rab11} \mathrm{(B)} \mathrm{at} \mathrm{the} \mathrm{Drosophila}$ NMJ. Single confocal slices of NMJ boutons stained with the indicated antibodies. Scale bars, $5 \mu \mathrm{m}$. Cdc42 and Rab11 localize to small puncta within Nwk-defined periactive zones. C, Quantification of mean bouton number per muscle area for segment A3, muscle 6/7.D, Quantification of mean satellite bouton number per muscle area for segments A2-A3, muscle 4. E, Representative confocal projections of anti-Cpx staining from NMJs quantified in D. Scale bar, $20 \mu \mathrm{m} . y, w$ was used as a wild-type strain. ${ }^{*} p<$ $0.05,{ }^{* * *} p<0.005$. Raw data are tabulated in supplemental Table 1 , available at www.jneurosci.org as supplemental material.

namin and Wsp. Other F-BAR/SH3 family members have been postulated to link dynamin and Wsp by multimerization via their F-BAR domains (Itoh and De Camilli, 2006; Tsujita et al., 2006; Shimada et al., 2007), but endogenous complexes containing Wsp and dynamin have only been demonstrated for the F-BAR/ SH3 protein syndapin (Kessels and Qualmann, 2006). Nwk could thus be in a position to bring dynamin and Wsp together. We have not been able to coimmunoprecipitate endogenous Wsp and Nwk using our antibodies. However, we find that dynamin immunoprecipitates contain Nwk but not Wsp, suggesting that Nwk-SH3a may switch associations between dynamin and Wsp. Another interpretation is that Wsp and dynamin binding are restricted to separate populations of Nwk molecules, and that the $\mathrm{SH} 3$ a domain thus acts in two parallel biochemical pathways.

In vivo analysis reflects the complexity of these $\mathrm{SH} 3$ domain interactions. $\mathrm{SH} 3 \mathrm{a}$ and $\mathrm{SH} 3 \mathrm{~b}$ of Nwk have both separate and overlapping functions in regulating synaptic growth (Figs. 5, 6), perhaps reflecting the multivalent nature of interactions in the Nwk network. [In addition to binding Nwk, Dap160 binds to both dynamin (Roos et al., 1998) and to Wsp (Giot et al., 2003).] Furthermore, the fact that mutation of both $\mathrm{SH} 3$ domains together (Nwk$\mathrm{SH} 3 \mathrm{a}^{\star} \mathrm{b}^{\star}$ ) produces additional dominant effects suggests that a non-SH3 ligand of Nwk is inappropriately titrated away from its function after mutation of Nwk SH3 domains. An excellent candidate ligand is the membrane itself, because the Nwk F-BAR domain has the potential to bind to and tubulate phospholipid bilayers. Determining the specific order and regulation of F-BAR/SH3 domain protein interactions with competing $\mathrm{SH} 3$ domain ligands and with the membrane will be important for uncovering the molecular mechanisms of these proteins during endocytosis.

NMJ overgrowth with an excess of satellite boutons is a hallmark of endocytic mutants (Dickman et al., 2006). We show that Nwk interacts with the endocytic machinery and that $c d c 42$ and $n w k$ mutants exhibit overproliferation of satellite boutons. A prominent function of endocytosis in nerve terminals is the recycling of synaptic vesicles. However, nwk single mutants (Coyle et al., 2004) and cdc42; nwk double mutants (S. Saraswati, A. A. Rodal, and J. T. Littleton, unpublished results) show no detectable defect in endocytosis of synaptic vesicles. One interpretation of this result is that receptor endocytosis is more sensitive to perturbation than synaptic vesicle recycling. However, given the documented function of Cdc42 and Wsp in endosomes (see below), it is more likely that Nwk functions in a later step of endocytic traffic. Importantly, although the synaptic vesicle endocytosis defects in shi (dynamin) and dap160 reflect the function of these molecules in the internalization step of endocytosis, synaptic overgrowth in these mutants could arise from defects at later steps of endocytic traffic, because dynamin functions in a variety of membranetrafficking events, ranging from Golgi traffic to endosome traffic (van Dam and Stoorvogel, 2002; Kessels et al., 2006). 
The endosomal system is organized into subdomains defined by specific members of the Rab GTPase family and adopts distinct morphology and ultrastructure in different cell types. Thus, functionally conserved Rab subdomains provide a unifying approach to understanding structurally diverse membrane systems. Rab11 controls the function of the recycling endosome in directing traffic to the cell surface (Sönnichsen et al., 2000) and colocalizes with Nwk in periactive zones at the Drosophila NMJ [although it can occasionally be observed in larger puncta (Khodosh et al., 2006)]. Like $c d c 42$ and nwk mutants, rab11 mutants have a profound defect in synaptic growth, exhibiting excessive satellite boutons (Khodosh et al., 2006). Cdc42 and WASp have recently been implicated in recycling endosome function (Parsons et al., 2005; Balklava et al., 2007). Thus, periactive zones may be the synaptic representation of the recycling endosome, with Cdc42 and Nwk controlling actin polymerization-dependent traffic of signaling complexes at this Rab11-positive compartment. Whether Cdc42 functions as a signal-responsive element in this compartment or forms part of the constitutive machinery for membrane traffic remains uncertain.

The TGF- $\beta$ /BMP family member Gbb (Marqués and Zhang, 2006; Collins and Diantonio, 2007) activates downstream signals that may be the critical targets of Nwk/Cdc42-mediated endocytosis in synaptic growth (Fig. 1). Indeed, recent work has shown that Gbb signaling is required for synaptic overgrowth in $n w k$ mutants, phosphorylation of the Gbb signaling target Mothers against decapentaplegic (Mad) is upregulated in $n w k$ mutants, and Nwk biochemically interacts with the intracellular domain of the Gbb receptor Tkv (O'Connor-Giles et al., 2008). However, other signaling pathways could equally be regulated by Nwk/ Cdc42-mediated endocytosis, lead to upregulation of phosphorylated Mad, and contribute to the synaptic overgrowth in $c d c 42$; $n w k$ mutants. One candidate pathway is the presynaptic component of the Wnt/Wg cascade, which may converge on Gbb/Mad regulation in the synapse as observed in other tissues (Fuentealba et al., 2007). We have not been able to detect any change in the steady-state localization of candidate cargoes in synaptic boutons in $n w k$ or $c d c 42$ mutants, suggesting that Nwk and Cdc42 are not required for the gross morphology of endosomes, but instead contribute to the rate of cargo trafficking through this compartment. Determining the specific signaling pathways, receptors, and their activation states in recycling endosomes will require tools to measure the activity and rates of traffic of specific receptors in situ.

Nwk is conserved from insects to higher vertebrates, and the mammalian genome encodes two Nwk homologs, which have not yet been characterized. However, Cdc42 and WASp-induced actin polymerization have been implicated in synapse formation in Aplysia sensory neurons and in mammalian hippocampal cultures (Udo et al., 2005; Shen et al., 2006). These reports suggest that the direct consequence of activating these proteins was the formation of filopodia that mature into synapses. An alternative hypothesis, consistent with the established function of Cdc42 and WASp family members in generating force for intracellular membrane traffic rather than in filopodial formation (Qualmann and Kessels, 2002; Biyasheva et al., 2004; Faix and Rottner, 2006; Kaksonen et al., 2006), is that synaptic growth regulatory functions of Cdc42 and WASp depend on endosomal traffic of signaling complexes by a similar mechanism to Drosophila Nwk-Wsp-induced synapse formation.

\section{References}

Abdul-Manan N, Aghazadeh B, Liu GA, Majumdar A, Ouerfelli O, Siminovitch KA, Rosen MK (1999) Structure of Cdc42 in complex with the GTPase-binding domain of the 'Wiskott-Aldrich syndrome' protein. Nature 399:379-383.

Balklava Z, Pant S, Fares H, Grant BD (2007) Genome-wide analysis identifies a general requirement for polarity proteins in endocytic traffic. Nat Cell Biol 9:1066-1073.

Ben-Yaacov S, Le Borgne R, Abramson I, Schweisguth F, Schejter ED (2001) Wasp, the Drosophila Wiskott-Aldrich syndrome gene homologue, is required for cell fate decisions mediated by Notch signaling. J Cell Biol 152:1-13.

Biyasheva A, Svitkina T, Kunda P, Baum B, Borisy G (2004) Cascade pathway of filopodia formation downstream of SCAR. J Cell Sci 117:837-848

Bouslama-Oueghlani L, Echard A, Louvard D, Gautreau A (2007) RNAi depleted Drosophila cell extracts to dissect signaling pathways leading to actin polymerization. J Biochem Biophys Methods 70:663-669.

Co C, Wong DT, Gierke S, Chang V, Taunton J (2007) Mechanism of actin network attachment to moving membranes: barbed end capture by N-WASP WH2 domains. Cell 128:901-913.

Collins CA, Diantonio A (2007) Synaptic development: insights from Drosophila. Curr Opin Neurobiol 17:35-42.

Coyle IP, Koh YH, Lee WC, Slind J, Fergestad T, Littleton JT, Ganetzky B (2004) Nervous wreck, an SH3 adaptor protein that interacts with Wsp, regulates synaptic growth in Drosophila. Neuron 41:521-534.

Dickman DK, Lu Z, Meinertzhagen IA, Schwarz TL (2006) Altered synaptic development and active zone spacing in endocytosis mutants. Curr Biol 16:591-598.

Duke-Cohan JS, Kang H, Liu H, Rudd CE (2006) Regulation and function of SKAP-55 non-canonical motif binding to the SH3c domain of adhesion and degranulation-promoting adaptor protein. J Biol Chem 281:13743-13750.

Faix J, Rottner K (2006) The making of filopodia. Curr Opin Cell Biol $18: 18-25$

Fazi B, Cope MJ, Douangamath A, Ferracuti S, Schirwitz K, Zucconi A, Drubin DG, Wilmanns M, Cesareni G, Castagnoli L (2002) Unusual binding properties of the $\mathrm{SH} 3$ domain of the yeast actin-binding protein Abp1: structural and functional analysis. J Biol Chem 277:5290-5298.

Fehon RG, Oren T, LaJeunesse DR, Melby TE, McCartney BM (1997) Isolation of mutations in the Drosophila homologues of the human Neurofibromatosis 2 and yeast CDC42 genes using a simple and efficient reverse-genetic method. Genetics 146:245-252.

Feng Y, Ueda A, Wu CF (2004) A modified minimal hemolymph-like solution, HL3.1, for physiological recordings at the neuromuscular junctions of normal and mutant Drosophila larvae. J Neurogenet 18:377-402.

Ferraro E, Peluso D, Via A, Ausiello G, Helmer-Citterich M (2007) SH3Hunter: discovery of $\mathrm{SH} 3$ domain interaction sites in proteins. Nucleic Acids Res 35:W451-W454.

Fuentealba LC, Eivers E, Ikeda A, Hurtado C, Kuroda H, Pera EM, De Robertis EM (2007) Integrating patterning signals: Wnt/GSK3 regulates the duration of the BMP/Smad1 signal. Cell 131:980-993.

Genova JL, Jong S, Camp JT, Fehon RG (2000) Functional analysis of Cdc42 in actin filament assembly, epithelial morphogenesis, and cell signaling during Drosophila development. Dev Biol 221:181-194.

Giot L, Bader JS, Brouwer C, Chaudhuri A, Kuang B, Li Y, Hao YL, Ooi CE, Godwin B, Vitols E, Vijayadamodar G, Pochart P, Machineni H, Welsh M, Kong Y, Zerhusen B, Malcolm R, Varrone Z, Collis A, Minto M, et al. (2003) A protein interaction map of Drosophila melanogaster. Science 302:1727-1736.

Higgs HN, Pollard TD (2000) Activation by Cdc42 and PIP(2) of WiskottAldrich syndrome protein (WASp) stimulates actin nucleation by Arp2/3 complex. J Cell Biol 150:1311-1320.

Ho HY, Rohatgi R, Lebensohn AM, Le Ma, Li J, Gygi SP, Kirschner MW (2004) Toca-1 mediates Cdc42-dependent actin nucleation by activating the N-WASP-WIP complex. Cell 118:203-216.

Huntwork S, Littleton JT (2007) A complexin fusion clamp regulates spontaneous neurotransmitter release and synaptic growth. Nat Neurosci 10:1235-1237.

Itoh T, De Camilli P (2006) BAR, F-BAR (EFC) and ENTH/ANTH domains in the regulation of membrane-cytosol interfaces and membrane curvature. Biochim Biophys Acta 1761:897-912.

Itoh T, Erdmann KS, Roux A, Habermann B, Werner H, De Camilli P (2005) 
Dynamin and the actin cytoskeleton cooperatively regulate plasma membrane invagination by BAR and F-BAR proteins. Dev Cell 9:791-804.

Kaksonen M, Toret CP, Drubin DG (2006) Harnessing actin dynamics for clathrin-mediated endocytosis. Nat Rev Mol Cell Biol 7:404-414.

Kessels MM, Qualmann B (2006) Syndapin oligomers interconnect the machineries for endocytic vesicle formation and actin polymerization. J Biol Chem 281:13285-13299.

Kessels MM, Dong J, Leibig W, Westermann P, Qualmann B (2006) Complexes of syndapin II with dynamin II promote vesicle formation at the trans-Golgi network. J Cell Sci 119:1504-1516.

Khodosh R, Augsburger A, Schwarz TL, Garrity PA (2006) Bchs, a BEACH domain protein, antagonizes Rab11 in synapse morphogenesis and other developmental events. Development 133:4655-4665.

Koh TW, Verstreken P, Bellen HJ (2004) Dap160/intersectin acts as a stabilizing scaffold required for synaptic development and vesicle endocytosis. Neuron 43:193-205.

Lnenicka GA, Keshishian H (2000) Identified motor terminals in Drosophila larvae show distinct differences in morphology and physiology. J Neurobiol 43:186-197.

Luo L, Liao YJ, Jan LY, Jan YN (1994) Distinct morphogenetic functions of similar small GTPases: Drosophila Drac1 is involved in axonal outgrowth and myoblast fusion. Genes Dev 8:1787-1802.

Marie B, Sweeney ST, Poskanzer KE, Roos J, Kelly RB, Davis GW (2004) Dap160/intersectin scaffolds the periactive zone to achieve high-fidelity endocytosis and normal synaptic growth. Neuron 43:207-219.

Marqués G, Zhang B (2006) Retrograde signaling that regulates synaptic development and function at the Drosophila neuromuscular junction. Int Rev Neurobiol 75:267-285.

Moseley JB, Maiti S, Goode BL (2006) Formin proteins: purification and measurement of effects on actin assembly. Methods Enzymol 406:215-234.

O’Connor-Giles KM, Ho LL, Ganetzky B (2008) Nervous Wreck interacts with Thickveins and the endocytic machinery to attenuate retrograde BMP signaling during synaptic growth. Neuron 58:507-518.

Parnas D, Haghighi AP, Fetter RD, Kim SW, Goodman CS (2001) Regulation of postsynaptic structure and protein localization by the Rho-type guanine nucleotide exchange factor dPix. Neuron 32:415-424.

Parsons M, Monypenny J, Ameer-Beg SM, Millard TH, Machesky LM, Peter M, Keppler MD, Schiavo G, Watson R, Chernoff J, Zicha D, Vojnovic B, $\mathrm{Ng} T$ (2005) Spatially distinct binding of Cdc42 to PAK1 and N-WASP in breast carcinoma cells. Mol Cell Biol 25:1680-1695.

Qualmann B, Kessels MM (2002) Endocytosis and the cytoskeleton. Int Rev Cytol 220:93-144.

Roos J, Kelly RB (1998) Dap160, a neural-specific Eps15 homology and multiple SH3 domain-containing protein that interacts with Drosophila dynamin. J Biol Chem 273:19108-19119.

Roos J, Kelly RB (1999) The endocytic machinery in nerve terminals surrounds sites of exocytosis. Curr Biol 9:1411-1414.

Shen W, Wu B, Zhang Z, Dou Y, Rao ZR, Chen YR, Duan S (2006) Activityinduced rapid synaptic maturation mediated by presynaptic Cdc42 signaling. Neuron 50:401-414.

Shimada A, Niwa H, Tsujita K, Suetsugu S, Nitta K, Hanawa-Suetsugu K, Akasaka R, Nishino Y, Toyama M, Chen L, Liu ZJ, Wang BC, Yamamoto M, Terada T, Miyazawa A, Tanaka A, Sugano S, Shirouzu M, Nagayama $\mathrm{K}$, Takenawa T, et al. (2007) Curved EFC/F-BAR-domain dimers are joined end to end into a filament for membrane invagination in endocytosis. Cell 129:761-772.

Sone M, Suzuki E, Hoshino M, Hou D, Kuromi H, Fukata M, Kuroda S, Kaibuchi K, Nabeshima Y, Hama C (2000) Synaptic development is controlled in the periactive zones of Drosophila synapses. Development 127:4157-4168.

Sönnichsen B, De Renzis S, Nielsen E, Rietdorf J, Zerial M (2000) Distinct membrane domains on endosomes in the recycling pathway visualized by multicolor imaging of Rab4, Rab5, and Rab11. J Cell Biol 149:901-914.

Stradal TE, Scita G (2006) Protein complexes regulating Arp2/3-mediated actin assembly. Curr Opin Cell Biol 18:4-10.

Tomasevic N, Jia Z, Russell A, Fujii T, Hartman JJ, Clancy S, Wang M, Beraud C, Wood KW, Sakowicz R (2007) Differential regulation of WASP and N-WASP by Cdc42, Rac1, Nck, and PI $(4,5) \mathrm{P} 2$. Biochemistry 46:3494-3502.

Tsujita K, Suetsugu S, Sasaki N, Furutani M, Oikawa T, Takenawa T (2006) Coordination between the actin cytoskeleton and membrane deformation by a novel membrane tubulation domain of $\mathrm{PCH}$ proteins is involved in endocytosis. J Cell Biol 172:269-279.

Udo H, Jin I, Kim JH, Li HL, Youn T, Hawkins RD, Kandel ER, Bailey CH (2005) Serotonin-induced regulation of the actin network for learningrelated synaptic growth requires Cdc42, N-WASP, and PAK in Aplysia sensory neurons. Neuron 45:887-901.

van Dam EM, Stoorvogel W (2002) Dynamin-dependent transferrin receptor recycling by endosome-derived clathrin-coated vesicles. Mol Biol Cell 13:169-182.

Wagh DA, Rasse TM, Asan E, Hofbauer A, Schwenkert I, Dürrbeck H, Buchner S, Dabauvalle MC, Schmidt M, Qin G, Wichmann C, Kittel R, Sigrist SJ, Buchner E (2006) Bruchpilot, a protein with homology to ELKS/ CAST, is required for structural integrity and function of synaptic active zones in Drosophila. Neuron 49:833-844.

Wang X, Shaw WR, Tsang HT, Reid E, O’Kane CJ (2007) Drosophila spichthyin inhibits BMP signaling and regulates synaptic growth and axonal microtubules. Nat Neurosci 10:177-185. 